\title{
HYBRID MIMO TRANSCEIVER SCHEME WITH ANTENNA ALLOCATION AND PARTIAL CSI AT TRANSMITTER SIDE
}

\author{
W. C. Freitas Jr. \\ Nokia Technology Institute \\ Brasília-DF, Brazil \\ ext-walter.freitas@nokia.com
}

\author{
F. R. P. Cavalcanti \\ Wireless Telecom Research Group \\ Fortaleza-CE, Brazil \\ rodrigo@gtel.ufc.br
}

\author{
R. R. Lopes \\ Communications Department \\ Campinas-SP, Brazil \\ rlopes@decom.fee.unicamp.br
}

\begin{abstract}
Hybrid multiple-input multiple-output (MIMO) transceiver scheme (HMTS) combines transmit diversity and spatial multiplexing, thus achieving at the same time the two possible spatial gains offered by MIMO channels. In the design of HMTS spatial diversity and spatial multiplexing branches are disposed in parallel in order to achieve diversity and multiplexing gains at the same time. Since the spatial multiplexing branches have no protection, they are more susceptible to the fading effect becoming the bottleneck in the performance of the whole transceiver. In this paper, we propose a solution to this bottleneck in the hybrid MIMO transceiver scheme using a partial channel state information at the transmitter side. The idea is to perform an antenna allocation. Thus, the most powerful subchannels are allocated to the most susceptible layers (spatial multiplexing branches). Through this solution we decrease the performance imbalance between the two layers of the HMTS G3+1, increasing the whole transceiver performance with low complexity feedback requirements.
\end{abstract}

\section{INTRODUCTION}

The use of the multiple antennas has proliferated now in wireless systems as a possible solution to the capacity limitation of the current wireless systems. With the use of multiple antennas over certain scenarios we can achieve an increase in the capacity almost linear with the number of antennas [1]. The idea is that the use of multiple antennas creates a multiple-input multiple-output (MIMO) linear system in which the MIMO channel linking the transmitter and receiver antennas can be seen as multiple single-antenna subchannels with no additional power consumption, time transmission and bandwidth. These multiple subchannels can be separated through their spatial signatures in an environment rich in multipaths. Another well-known advantage of multiple antennas is in providing spatial diversity through the multiple links created by the multiple antennas. The idea is that with multiple links there exists a lower probability that all of them experiment deep fading.

More specifically, if there are $M$ transmit and $N$ receive antennas, generically denoted as $(M \mathrm{Tx}-N \mathrm{Rx})$, with sufficient signal scattering and antenna spacing, there are $M N$ independent links between the transmitter and the receiver. In this situation it is possible to provide an $M N$-fold protection against channel fading. This protection is called diversity gain and the number of independent links is the diversity order. On the other hand, there are $\min (M, N)$ degrees of freedom, which can be used to spatially multiplex data for increase spectral efficiency. This gain in multiplexing symbols through the MIMO wireless channel is known as spatial multiplexing gain.

MIMO schemes are known to provide these two main types of gains: spatial multiplexing gain and diversity gain. Spatial multiplexing gain describes the higher data rates that can be obtained using the spatial subchannels created by the MIMO channel. An example of a pure multiplexing scheme is the vertical Bell laboratories layered space-time (VBLAST) [1]. On the other hand, pure diversity schemes, like space-time block codes (STBC) [2, 3], are concerned with diversity gain. In other words, their objective is to increase the link reliability against fading. Apart from these two gains, it is also possible to achieve coding gain, as in the case of space-time trellis codes (STTC). This topic will be left to a future investigation. In this article, we focus on the spatial multiplexing and diversity gains.

It was shown in [4] that there exists a tradeoff between these two gains in the MIMO wireless channel. Zheng and Tse have shown that when one tries to maximize one possible gain of the MIMO wireless channels, this leads to a degradation of the other gain. For example, space-time block codes (STBC), well-known schemes in providing diversity gain, have no concerns about the capacity gain. On the other hand, vertical Bell laboratories layered space-time (VBLAST) schemes were designed aiming multiplexing as many different symbols as possible, but does not provide any diversity gain.

Zheng and Tse just characterized the tradeoff, not proposing any scheme capable of achieving it. A solution in this direction was proposed with a modification in the VBLAST scheme, called Diagonal BLAST [1], in which the transmitted symbols are multiplexed in all the transmit antennas available, but in different time instants. Unfortunately, this solution brings a considerable delay in order to achieve a diversity gain, and thus is not very practical.

Tarokh et al. in [5] presented the idea of combining array processing in the receiver and space-time coding strategies through multiple transmit antennas to reach at the same time reliable and very high data rate communication. Here we present a similar structure to that in [5], denoted Hybrid MIMO transmission schemes (HMTS). HMTS arise as a solution to jointly achieve spatial multiplexing and diversity gains. With HMTS, it is possible to considerably increase the data rate while keeping a satisfactory link quality in terms of bit error rate (BER). In fact, HMTS apply pure diversity schemes (e.g. STBC) jointly with pure spatial multiplexing schemes (e.g. VBLAST), so that parts of the data are space-time coded across 


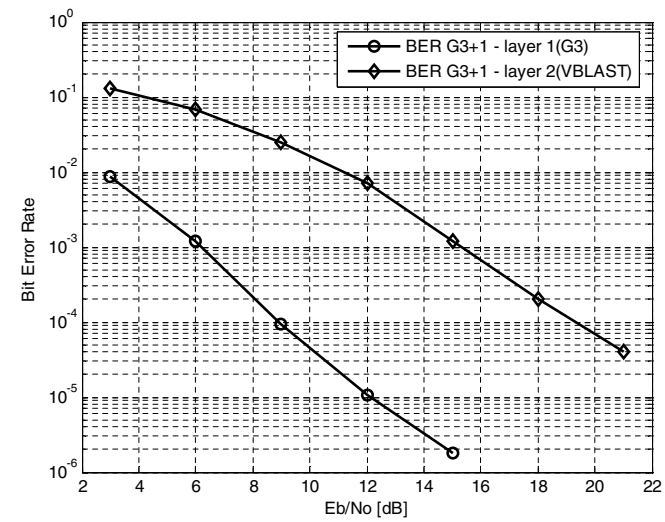

Figure 1: Bit error rate performance comparison for layers of the HMTS G3+1.

some antennas, and these parts are combined in layers, using a VBLAST approach. As spatially-multiplexed layers see each other as interference, interference cancellation (IC) similar to that employed in VBLAST is mandatory in the receiver.

Since the spatial multiplexing branches are transmitted with no protection and are thus more susceptible to fading, these branches constitute a bottleneck in the performance. We can observe this bottleneck looking at Fig. 1, which shows a comparison between the two branches (diversity and multiplexing) of the HMTS called G3+1 [6]. In this figure we can see the huge performance imbalance between the spatial diversity branch (layer 1 - STBC G3) and the spatial multiplexing branch (layer 2 - VBLAST).

Through antenna allocation for the VBLAST layers, we propose a solution to the bottleneck. This allocation requires a partial channel state information (CSI) at the transmitter side, which in turn require little feedback from the transmitter. And as IC algorithm we consider an efficient structure that performs IC in a two-stage approach, maintaining the low complexity of the STBC decoder receivers.

This article is organized as follows. In section II, we present the MIMO system and channel model. In Section III we propose the HMTS considered in this work. Section IV presents performance results and section VI states some conclusions and anticipate future work.

\section{System AND Channel Models}

In this paper, we consider a transmitter equipped with an $M$-element antenna array and a receiver equipped with an $N$-element antenna array. The transmitted signals are assumed to go through a random channel matrix $\mathbf{H}$. The wireless channel is assumed to have rich-scattering and flat-fading. The fading between each transmit and receive antenna pair is assumed to be independent and the entries of $\mathbf{H}$ are circularly symmetric Gaussian random variables. The quasi-static block fading model is assumed; in other words, the channel matrix $\mathbf{H}$ is randomly generated, but remains constant during the transmission of one space-time code word of length $K$. A new random channel matrix, independent of the previous one, is then generated for each new space-time code word. We disregard the frequency selectivity of the channel since it is well-known that a frequency selective channel can be converted into parallel flat-fading channels by inverse fast Fourier transform (IFFT) and fast Fourier transform (FFT). Thus, considerations presented here are also applicable to frequency selective channels. Furthermore, we assume i.i.d circularly symmetric Gaussian noise samples. For all the MIMO transmission schemes, we assume that the total transmit power is fixed (normalized to 1) and equally divided across the transmit antennas. Ideal symbol timing is assumed at the receiver. Thus, we can relate the transmit and receive symbols through the relation at time $k$ in complex baseband form and at the symbol rate

$$
\mathbf{x}[k]=\sqrt{\frac{\rho}{M}} \mathbf{H}[k] \mathbf{s}[k]+\mathbf{v}[k],
$$

where $\mathbf{x} \in \mathcal{C}^{N}$ denotes the vector of complex received symbols during any given channel use, $\mathbf{s} \in \mathcal{C}^{M}$ denotes the vector of the complex transmitted symbols, $\mathbf{H} \in \mathcal{C}^{N \times M}$ denotes the channel matrix, $\mathbf{v} \in \mathcal{C}^{N}$ is the zero-mean, unit variance and complex-Gaussian distributed noise that is spatially and temporally white, and $\rho$ is the signal-to-noise ratio (SNR).

\section{Combining Array Processing And Space-Time CODES}

In this section, we will present two transmission structures that combines the robustness of space-time codes to the high rates of VBLAST. In other words, we will present structures that achieve both diversity and multiplexing gains. The basic idea behind both structures is to combine array processing and space-time coding, as first presented in [5].

In [5] Tarokh et al. combined space-time trellis codes (STTC) and array processing by partitioning antennas at the transmitter into small groups. The signal transmitted in each group of antennas goes through a given STTC, called by authors as component codes. At the receiver, the signals from each STTC are separated by a linear processing technique that suppresses signals transmitted from other groups of antennas, by treating them as interference. Then, the STTC are individually decoded.

Tarokh's et al. idea involves a fixed transmission structure, and adapting the transmitter to the channel conditions was not considered. However, since the wireless channel is random, using a fixed structure in some cases could represent a loss of the resources. We now present a structure similar to that in [5]. This structure is called HMTS [6] and it also aims to achieve at the same time diversity and multiplexing gains. However, in our case we consider a family of transmission structures for three and four transmit antennas that are capable to reach this goal. Thus, our approach is naturally an adaptable structure, changing the focus in accordance to the MIMO channel characteristics [6,7]. Another difference between our approach and that presented in [5] is that we consider STBC instead STTC. 


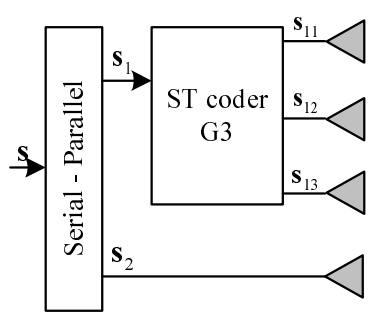

Figure 2: Structure of the HMTS G3+1 transmitter

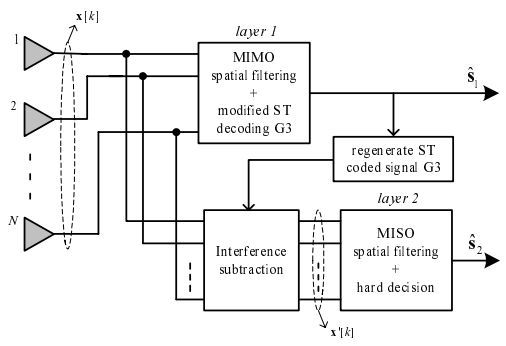

Figure 3: Structure of the HMTS G3+1 receiver.
Through the general framework of LDC [8] it is possible to represent a vast set of MIMO transceiver structures, including the HMTS proposed here and the proposed by Tarokh. Next, we describe the HMTS considered in this work.

\section{A. Hybrid MIMO Transceiver Structure:G3+1}

In general, the transmission process of a HMTS can be divided in layers, extending the definition of a layer in VBLAST. In the hybrid case, a layer consists of the streams of symbols at the output of a STBC, which are sent to a group of antennas, or to an uncoded stream, which is transmitted from a single antenna. Based on this concept of layers, HMTS combine pure diversity schemes (e.g. STBC) with pure spatial multiplexing schemes (e.g. VBLAST). In HMTS, some layers are space-time coded across two, three or four antennas. For the remaining layers, a VBLAST approach is used. With this idea, HMTS achieve a compromise between spatial multiplexing and transmit diversity gains.

In the remainder of this section we present one specific HMTS, but other combinations are possible [6]. The notation consider is, the space-time coded layers follow the denomination of the STBC used (e.g. G2 or G3) [3], while each uncoded streams following the VBLAST scheme is denoted in the label of HMTS as (+1). For example, the system designed to four transmit antennas consisting of two layers, one space-time coded through the G3 scheme and other uncoded layer following the VBLAST scheme, is denoted as G3+1.

We now present one possible HMTS, called G3+1. This hybrid scheme, whose structure is shown in Fig. 2, employs a 4-element transmit antenna array with two spatial multiplexing layers. A standard G3 space-time block code is used at the first layer. The other layer is not space-time-coded and follows the VBLAST idea. In the G3+1 scheme, the transmitted signals can be organized in a equivalent space-time coding matrix as described below

$$
\mathbf{S}_{G 3+1}[k, \ldots, k+7]=\left[\begin{array}{cccc}
s_{1} & s_{2} & s_{3} & s_{5} \\
-s_{2} & s_{1} & -s_{4} & s_{6} \\
-s_{3} & s_{4} & s_{1} & s_{7} \\
-s_{4} & -s_{3} & s_{2} & s_{8} \\
s_{1}^{*} & s_{2}^{*} & s_{3}^{*} & s_{9} \\
-s_{2}^{*} & s_{1}^{*} & -s_{4}^{*} & s_{10} \\
-s_{3}^{*} & s_{4}^{*} & s_{1}^{*} & s_{11} \\
-s_{4}^{*} & -s_{3}^{*} & s_{2}^{*} & s_{12}
\end{array}\right]
$$

where the spatial dimension varies column-wise and the temporal dimension row-wise. From (2), it can be seen that $K=12$ information symbols are transmitted in $T=$ 8 consecutive signaling intervals $([k, \ldots, k+7])$. Thus, the effective spectral efficiency of this scheme is equal to $(K / T) \cdot \log _{2} \mathcal{M}=1,5 \cdot \log _{2} \mathcal{M}$ bps/Hz, where $\mathcal{M}$ is the cardinality of the modulation scheme considered. This represents a $200 \%$ increase in data rate compared to other 4Tx MIMO transmission structures, like STBC G4 which achieves a spectral efficiency of $0,5 \cdot \log _{2} \mathcal{M}$ bps/Hz.

In a general way the MIMO transmission structures can be organized through a space-time equivalent matrix. The space-time equivalent matrix $\mathbf{S}$ defines the transmitted symbols in each antenna per signaling interval. Thus, the matrix $\mathbf{S}$ has dimension $T \times M$, where the columns represent the transmit antennas and the rows represent the signaling interval.

In [8], the authors presented codes that generalize all linear MIMO transmission structures, known as linear dispersion codes (LDC). Through LDC we can represent all linear MIMO space-time equivalent matrix $\mathbf{S}$ of the transmission structures (e.g. STBC, VBLAST-based structures and also the HMTS that combines the advantages of both, STBC and VBLAST).

\section{B. Modified Interference Cancellation Algorithm for the Hybrid MIMO Transceiver Schemes}

Since HMTS combine STBC and VBLAST in parallel, all HMTS have at least two layers, at least one of which is space-time block coded. Further, they all employ orthogonal STBC, whose maximum-likelihood detection involves simple linear operations in the receiver. We now propose a receiver for the HMTS that combines the simplicity of successive interference cancellation (SIC) [9] algorithm with the simplicity of decoding of orthogonal STBC. In fact, we adapt the IC algorithm in such a way that the orthogonal structure of the space-time code is preserved as much as possible in its output signal. The general structure of the receiver is shown in Fig. 3.

In the case of $\mathrm{G} 3+1$, we have two layers: a standard G3 STBC at the first layer and a non-space-time-coded layer. In Fig. 3 the MIMO spatial filter mitigates the interference from other layers, so that its output signal consists of a single space-time-coded signal or of a single uncoded stream. In our SIC approach, we will first detect the G3 layer, which is more robust. To that end, we first employ a linear minimum-mean-square-error (MMSE) filter to eliminate the interference from the uncoded layer. In this case, the error vector at the output of the MIMO-MMSE spatial filter is given 
by

$$
\mathbf{e}[k]=\mathbf{W} \mathbf{x}[k]-\mathbf{H}_{d} \mathbf{s}_{1}[k]=\mathbf{W} \mathbf{x}[k]-\mathbf{x}_{d}[k],
$$

where $\mathbf{x}_{d}[k]=\mathbf{H}_{d} \mathbf{s}_{1}[k]$ is now the desired space-time coded signal associated to the first multiplexing layer. This operation is depicted in Fig. 3.

Contrarily to the classical SIC where in the MIMO-MMSE spatial filter the desired signal is the transmitted signal $\mathbf{s}_{1}[k]$, here the desired signal consists of the original transmitted signal modified by desired MIMO channel response $\mathbf{H}_{d}$, which can be interpreted as the "virtual" channel between the transmitter and the output of the spatial filter. In this fact resides the main difference between the traditional SIC and this modified version.

The MSE cost function may be written as

$$
J_{M M S E}=E\left\{\left\|\mathbf{W} \mathbf{x}[k]-\mathbf{x}_{d}[k]\right\|^{2}\right\} .
$$

The optimal coefficients are found by minimizing the above cost function with respect to $\mathbf{W}$. The solution is given by

$$
\mathbf{W}=\mathbf{R}_{\mathbf{x}_{d} \mathbf{x}} \mathbf{R}_{\mathbf{x x}}{ }^{-1}
$$

where $\mathbf{R}_{\mathbf{x} \mathbf{x}}=E\left\{\mathbf{x}[k] \mathbf{x}^{H}[k]\right\}$ and $\mathbf{R}_{\mathbf{x}_{d} \mathbf{x}}=E\left\{\mathbf{x}_{d}[k] \mathbf{x}^{H}[k]\right\}$ are the input covariance matrix and a cross-correlation matrix, respectively.

Figure 3 shows the architecture of the modified SIC receivers for the HMTS G3+1. Clearly, we can see in this figure that the layers are processed successively, in a two stage process in which

1. first a nulling of the interference from the undetected layers is made, then, the output signal goes through a decoder for the STBC used in this layer;

2. finally, the received space-time coded signal corresponding to this layer is regenerated and its impact is cancelled from the received signal.

\section{Performance Results}

This section presents simulation results for the problem of uncoded layers for the hybrid MIMO transceiver schemes. We present the performance of the proposed solution in terms of bit error rate (BER). The performance of the HMTS is evaluated here by means of numerical results from Monte-Carlo simulations. The curves are plotted against the average $\mathrm{Eb} / \mathrm{No}$ per receive antenna. Perfect channel estimation is assumed ${ }^{1}$. Unless otherwise noted, all schemes employ binary-phase shift-keying (BPSK) modulation. We assume a MIMO system with 4 transmit and 3 receive antennas.

As defined before, the HMTS employs multiplexing layers in which some layers are space-time coded through an STBC and other layers are transmitted following the VBLAST approach. Since the layers following the VBLAST idea transmit with no protection, these layers form the bottleneck in the whole receiver performance. We can confirm this claim in Fig. 1,

\footnotetext{
${ }^{1}$ The degradation due to imperfect channel estimation is negligible if the number of transmit antennas is small [9], as is the present case.
}

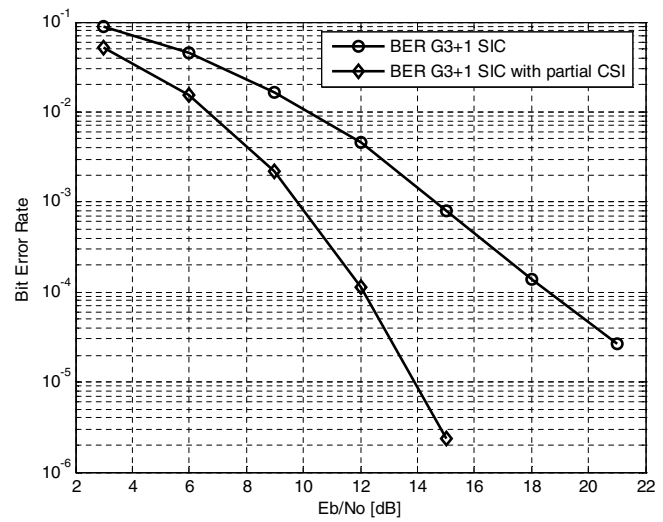

Figure 4: Result considering clever antenna allocation and partial CSI at transmitter side.

layer 1 (STBC G3) presents an excellent result in terms of BER, while layer 2 (VBLAST) has a huge difference in the performance.

In this work we consider a solution for systems where the transmitter has some knowledge of the CSI. In this case, the transmitter may make a clever layer allocation, in such a way that the better channel is allocated for the VBLAST layer, since this is the most susceptible to fading. In the next section, we describe in more details the proposed solution.

In this section we consider the idea of the transmit antenna selection scheme for the HMTS based on a partial CSI at the transmitter, this solution is similar to that presented in [10]. Since the bottleneck is in the VBLAST layers, the receiver estimates all the CSI and with this information obtain the power of each subchannel in an ordered way. Thus, just the order of the subchannels power is fedback to the transmitter and in this way the best subchannels for the VBLAST layers are selected.

Let the channel matrix linking each receiver and transmitter antenna be represented as

$$
\mathbf{H}_{\mathrm{Rx}}=\left[\begin{array}{cccc}
h_{11} & h_{12} & h_{13} & h_{14} \\
h_{21} & h_{22} & h_{23} & h_{24} \\
\vdots & \vdots & \vdots & \vdots \\
h_{N 1} & h_{N 2} & h_{N 3} & h_{N 4}
\end{array}\right] .
$$

From the transmitter point of view we can define also a channel matrix $\mathbf{H}_{\mathrm{Tx}}$ that is related with $\mathbf{H}_{\mathrm{Rx}}$ through

$$
\mathbf{H}_{\mathrm{Tx}}=\mathbf{H}_{\mathrm{Rx}}^{T} \text {. }
$$

This new matrix has dimension $4 \times N$ and each line represent the links between each transmitter antenna and all the receiver ones as

$$
\mathbf{h}_{m}=\left[\begin{array}{llll}
h_{m 1} & h_{m 2} & \ldots & h_{m N}
\end{array}\right], m=1, \ldots, M .
$$

In this way the power of each transmitter link can be obtained as

$$
P_{\mathbf{h}_{m}}=\left(\mathbf{h}_{m} \mathbf{h}_{m}^{H}\right)
$$

If the transmitter knows the order of the links, it can use the strongest transmitters subchannles to transmit VBLAST layers, 


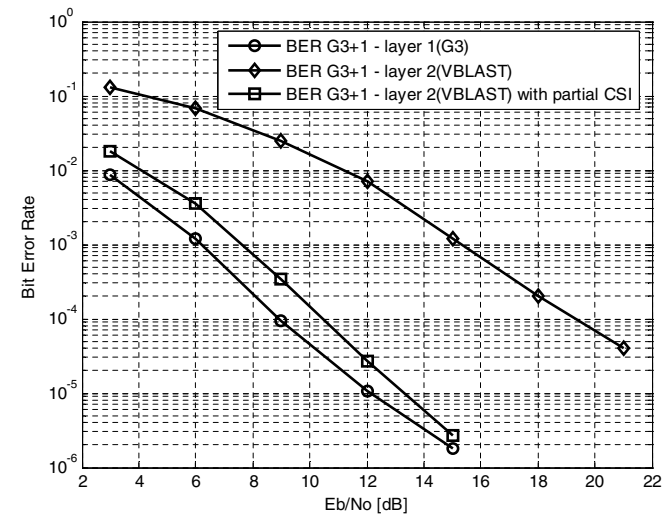

Figure 5: Result per layer considering clever antenna allocation and partial CSI at transmitter side.

and the others are left to transmit using the STBC scheme. This provides some extra protection to the VBLAST layers. An interesting point is that is not necessary that the transmitter knows all the CSI, but just the order of the more powerful links. We denote this approach as partial CSI. Thus, no large overhead is created, and just some bits could be used to carry this information to transmitter.

Figure 4 shows the performance of the whole receiver when partial CSI is known at the transmitter. Thus, subchannels power is fedback to the transmitter and in this way the best subchannels for the VBLAST layers are selected. The performance of the HMTS G3+1 regarding SIC with partial CSI achieves a huge gain, about $6 \mathrm{~dB}$ for a BER of $10^{-4}$, compared with the case where there is no antenna allocation.

Figure 5 shows the same comparison but per layer. Since the HMTS G3+1 has two layers:

1. layer 1(G3) - a standard G3 space-time block code;

2. layer 2(VBLAST) - layer 2 is not space-time-coded and follows the VBLAST idea.

In Fig. 5, our benchmark is the performance of the layer 1 space-time coded through the G3 STBC scheme. We can see that using the partial CSI at the transmitter the performance of the VBLAST layer is close to that of the G3 layer. Thus, the imbalance between the two layers of the HMTS G3+1 is drastically decreased. In fact, we observe a gain of around $9 \mathrm{~dB}$ between the VBLAST layers with and without partial CSI. Even, with partial CSI and antenna allocation the layer 2 of the HMTS G3+1 does not outperforms the layer $1(\mathrm{G} 3)$. This is due to the higher diversity order of the layer 1. STBC schemes could decode their symbols with just one receiver antenna, in this situation we are considering 3 receiver antennas. This explains why layer $1(\mathrm{G} 3)$ outperforms layer 2(VBLAST) with partial CSI.

\section{CONCLUSiOnS}

In this work we evaluated the performance of a possible solution for the bottleneck present in the HMTS G3+1.
The partial CSI at transmitter side provides a good result, decreasing the performance difference between the layers of the HMTS requiring a simple parameter to be estimated at receiver and just the order of the strengths of each subchannels need to be fedback to the transmitter. As perspectives, we mention the comparison when there is spatial correlation in the receiver side, as well as a performance evaluation under other MIMO channel models.

\section{REFERENCES}

[1] G. J. Foschini, "Layered Space-Time Architecture for Wireless Communications in a Fading Environment when using Multiple Antennas," Bell Labs Tech. J., v.1, n.2, pp.41-59, 1996.

[2] S. Alamouti, "A simple transmit diversity technique for wireless communications," IEEE Journal of Selected Areas in Communications, vol.16, pp.1451-1458, Oct 1998.

[3] V. Tarokh, H. Jafarkhani and A. R. Calderbank , "Space-time block codes from orthogonal designs," IEEE Transactions on Information Theory, vol. 5, pp. 1456-1467, July 1999.

[4] L. Zheng and D. Tse, "Diversity and Multiplexing: A Fundamental Tradeoff in Multiple Antenna Channels," IEEE Transactions on Information Theory, vol. 49, pp. 1073-96, May 2003.

[5] V. Tarokh, A. Naguib, N. Seshadri and A. R. Calderbank, "Combined Array Processing and Space-Time Coding," IEEE Transactions on Information Theory, vol. 45, pp. 1121-1128, May 1999.

[6] W. C. Freitas Jr., et al., Performance of MIMO Antenna Systems with Hybrids of Transmit Diversity and Spatial Multiplexing Using Soft-Output Decoding. Lecture Notes in Computer Science, Springer-Verlag Heidelberg, v.3124, p.28-37, Aug. 2004.

[7] W. C. Freitas Jr., F. R. P. Cavalcanti, A. L. F. de Almeida and R. R. Lopes, "Exploiting Dimensions of the MIMO Wireless Channel: Multidimensional Link Adaptation," IEEE VTC Spring Stockolm-Sweden, 2005.

[8] B. Hassibi and B. M. Hochwald, "High-rate codes that are linear in space and time," IEEE Transactions on Information Theory, vol. 48, pp. 1804-1824, July 2002.

[9] B. Vucetic and J. Yuan, "Space-time coding," West Sussex, England; Hoboken, NJ : Wiley, c2003. TK5102.92.V82, 2003.

[10] D. Gore and A. Paulraj, "MIMO antenna subset selection for space-time coding", IEEE Trans. Sig. Proc., vol.50, pp. 2580-2588, Oct. 2002. 